\title{
BOUNDARY MAINTENANCE IN LIVING SYSTEMS THEORY AND SOCIAL ENTROPY THEORY
}

\author{
Kenneth D. Bailey ${ }^{1}$ \\ ${ }^{1}$ University of California, Los Angeles, CA, USA, Kbailey@soc.ucla.edu
}

\begin{abstract}
Past analyses of social systems have too often relied upon obsolete dualities such as open system/closed system. The purpose of this paper is to provide a more comprehensive analysis of the full range of boundary-maintenance operations that are at work in the modern complex social system. I first analyze the role of the boundary in Living Systems Theory, and then turn to the analysis and comparison of boundary maintenance in Social Entropy Theory. Among the topics discussed are boundary control for matter-energy flows, boundary control for information flows, types of information inflows across social-system boundaries, types of information outflows across social-systems boundaries, and types of matter-energy inflows and matterenergy outflows across social systems boundaries. Among the types of information inflows discussed are neutral, fatal, catalytic, and canceling. Among the types of information outflows discussed are obligatory, routine, and formatted. And finally, some of the matter-energy inflows and outflows discussed are obligatory, optional, and routine.
\end{abstract}

Keywords: boundary, living systems theory, social entropy theory, matter-energy flow, information flows

\section{INTRODUCTION}

The boundary has special significance in systems theory as it, by definition, separates the system from its environment. In so doing, it effectively defines and operationalizes the system. The significance of the boundary for both intra-system relations and inter-system relations cannot be overstated. The principles of selfreference (see Luhmann, 1995; Bailey, 1997) are clearly relevant for boundary analysis in the sense that once a boundary is established, the system references that boundary in virtually all of its future actions, both internal and external.

Unfortunately, the social-systems literature has traditionally impeded discussion of the boundary through its use of the open system/closed system duality (Bailey, 1990, 1994). The situation has improved somewhat in recent years as theorists such as Luhmann (1995) have adapted autopoietic theory, which views the system as simultaneously open and closed, remaining organizationally closed, while being open to exchanges across system boundaries. However, the role of the boundary in the modern complex social system is far more complex that even the autopoietic literature recognizes. It is imperative that the boundary be viewed in a more comprehensive and complex fashion, and this is the goal fo the present paper. This will be done by first comparing the role of the boundary in both Miller's (1978) Living systems Theory (LST), and Bailey's (1990, 1994) Social Entropy Theory (SET), and then 


\section{Boundary Maintenance in LST and Social Entropy Theory}

moving to a more exhaustive analysis of the boundary actions involved in maintaining adequate flows across social-system boundaries.

\section{THE BOUNDARY IN LST}

Living Systems Theory (Miller, 1978, Miller and Miller, 1992) is based upon the analysis of 20 critical subsystems for each of eight hierarchical levels. These levels are the cell, organ, organism, group, organization, community, society, and supranational system. The 20 critical subsystems comprise two subsystems that are said to process both matter-energy and information, eight that process matter-energy only, and 10 that process information only. The two subsystems that process both matter-energy and information are the boundary and the reproducer. The eight subsystems that process only matter-energy are the ingestor, distributor, converter, producer, matter-energy storage, extruder, motor, and supporter. The 10 subsystems that process information only are the input transducer, the internal transducer, the channel and net, the decoder, the associator, the memory, the decider, the encoder, the output transducer, and the timer.

The extreme importance of the boundary, and its role in the self-reference of the living system, is revealed through only cursory perusal of the eight subsystems that process matter-energy and the 10 subsystems that process information. For example, Miller's (1978, p. 3) definition of the ingestor explicitly includes the boundary, by defining the ingestor as "the subsystem which brings matter-energy across the system boundary from the environment". Thus, without knowledge of the boundary, one would be unable to define or describe the ingestor or the remaining seven matterenergy processing subsystems. Miller $(1978$, p. 3) refers indirectly to the boundary in five instances, by referring to inputs (the distributor converter, and producer) or outputs (the producer and extruder) or the environment (the motor). Although he does not use the term "boundary" in these definitions, the terms that he does use (input, output, and environment) cannot be defined without a boundary. The only two matter-energy subsystems which are defined solely as internal entities without any direct or indirect reference to a boundary are the matter-energy storage subsystem, and the supporter. However, the definitions of these two entities both refer to the system, whose very definition depends upon the existence of the boundary.

A similar degree of reliance on the boundary is seen in the definitions of the information-processing subsystems (Miller, 1978, p. 3). For example, the input transducer and the decoder both are defined in terms of inputs into the system across the boundary, while the encoder and the output transducer depend on the notion of outputs across the boundary. This leaves the internal transducer, channel and net, associator, memory, decider, and timer as primarily internal subsystems, with little direct reference to the boundary.

\section{THE BOUNDARY IN SET}

Social Entropy Theory (Bailey, 1990, 1994) views the social system as an entity that seeks to maintain internal entropy at some optimal level below maximum entropy. The role of the boundary is crucial in this task. The societal boundary must be able to 


\section{Boundary Maintenance in LST and Social Entropy Theory}

open in the proper manner and at the proper time to ensure that adequate inputs of energy and information can be attained from the environment, that refuse or harmful material can be properly extruded from the system, and that needed exports of matterenergy and information can be moved across the boundary.

Conversely the boundary must be able to close in the proper manner and at the proper time in order to ensure that the system remains secure from threats outside in the environment (prohibits harmful inputs), and also guards against the loss of valuable internal resources (prohibits harmful outflows). Thus, the functions of boundary maintenance are fourfold: to open in a manner that (1) ensures needed inputs (and (2) ensures needed outputs, and to close in a manner that (3) prohibits harmful inputs and (4) prohibits loss of valuable matter-energy or information through harmful outputs.

Social Entropy Theory states that the social system functions on a daily basis by regulating six key system components: Population (P), Information (I), Space (S), Technology (T), Organization (O), and Level of Living (L), which can be remembered by the alternative acronyms of PILOTS or PISTOL. This framework can be used to analyze the large variety of flows across the body of a given social system.

These flows across social boundaries can be roughly divided into matter-energy flows, or information flows (Miller, 1978). We can identify eight essential dimensions presented in that can be represented in terms of flows or movement across boundaries. These are: (1) human population; (2) elements from the internal environment (for example, plants, animals, or minerals); (3) energy; (4) culture; (5) technology; (6) information; (7) organization; and (8) level of living (for example, wealth).

It seems unnecessary at this point to specify all of the potential boundary processes for all eight of these types of flows. It should suffice now to present a table (Table 1) showing the boundary-maintenance processes for both matter-energy flows and information flows. This table is meant to be generic, meaning that all 36 possibilities (18 for energy and 18 for information) may occur at all hierarchical levels (society, group, and so forth), and in all types of societies.

Table 1. Models of Boundary Control for Matter-Energy and Information Flows Across Group Boundaries

\begin{tabular}{lll}
\hline Model \# & Inputs (Imports) & Outputs (Exports) \\
\hline 1 (closed system) & Always Closed & Always Closed \\
2 & Always Open & Always Closed \\
3 & Always Closed & Always Open \\
4 (open system) & Always Open & Always Open \\
5 & Always Closed & Variable \\
6 & Variable & Always Closed \\
7 & Always Open & Variable \\
8 & Variable & Always Open \\
9 & Variable & Variable \\
& &
\end{tabular}




\section{Boundary Maintenance in LST and Social Entropy Theory}

Social groups obviously can vary widely in their ability to achieve their specific boundary maintenance goals. Efficacy in boundary control can depend upon a number of factors, including the following.

1. The distance from the central decider (government) to the boundary.

2. Accessibility of the boundary.

3. Physical conditions, terrain, and topography at the boundary.

4. Length of the boundary.

5. Form of the boundary, and so forth.

The physical form of the boundary can vary widely. In some cases it may be basically only an "informational" boundary consisting of a line on the ground, or merely a sign indicating the location of the boundary. In other cases the boundary might be a wire, or a small fence, or a very large and impenetrable fence or wall such as the Berlin Wall. In some cases it is unguarded. In other the boundary is an expanse of territory that is heavily guarded and filled with land mines, such as the North Korean-South Korean De-Militarized Zone (DMZ). Although it is a gross generalization, we can say that the boundaries that control the flow of matter-energy tend to be the more traditional physical boundaries such as gates, fences, and steel shutters. These gates and shutters are often mechanized, and can thus be controlled through motors that are capable of moving them rather rapidly.

Notice, however, that even physical boundaries that are regulated to either bar or admit physical (matter-energy) flows, also require the use of information. Thus, boundary regulation is generally not solely accomplished through physical (matterenergy) processing or solely through information-processing, but is a combination of both.

For example, consider the common occurrence of visitors approaching the gate of a walled mansion in a wealthy residential area. Assume that this is the front gate, and is deemed a "public entrance". Assume also that it is never used for "transducer" functions such as garbage removal (another gate, in the rear, serves this purpose), nor for exits of persons from the mansion (yet another gate, a side exit gate, serves this function). Thus, in Table 1, the front gate represents Model number 6. It is always closed to outputs, and it is variable with regard to its openness of input. That is, it is not permanently closed to input, and so does not fit Model number 1, but neither is it permanently open, so it does not fit Model number 2. Thus, of the three models are that always closed to outputs (Models number 1,2, and 6), number 6 is the only one that fits this example.

Notice however, that while the gate is a matter-energy (physical) barrier, and is regulating the flow of matter-energy (primarily people), this regulation ultimately depends not only on matter-energy, but also on information. After the people approach the gate, the gate will remain closed, and they will not be allowed to enter unless some information is received by the decider that indicates that entry would be warranted, and would not be harmful to the group (family) residing in the mansion. This information must be transmitted by some form, such as direct voice transmission (speaking) wireless voice transmission, wired voice transmission, video, and so forth.

The length or distance of the information transmission required for gate regulation may vary widely, from a short-distance transmission to a gate-keeper such as an on- 


\section{Boundary Maintenance in LST and Social Entropy Theory}

site guard stationed at the gate, to a much longer distance transmission to some more remote gate-keeping location. The particular information to be transmitted needs to be sufficient as a signal either to admit, or not admit.

This can be determined in a number of ways, such as the clothing of the persons desiring entry, an identification badge, or credentials (Miller, 1978), passports, passwords, and so forth. Since all of these are increasingly easy to imitate and thus to falsify, security decisions will increasingly be determined through data that is more difficult to alter. This includes biometric data such as fingerprints, unique bloodvessel patterns in the face, patterns in the eyes, or even ultimately through rapid DNA determination conducted at the site.

While information is always required, along with matter-energy to process the admittance or barring of entry of matter-energy at a physical barrier, the reverse is also true. That is, the decision to either admit or bar information generally also necessitates the utilization not only of information, but also of some matter-energy or physical apparatus ("hardware"). That is, while information may be transmitted in via a "wireless" device, at some point, generally the organization and decoding of the message (and perhaps in between, as in the case of a satellite or transmission tower) there is "hardware" (matter-energy). You may devise a password to determine whether the information is to be admitted, and you may consider this password to be "information" rather than a physical entity, or matter-energy. Nevertheless, unless you imbed this information in some form of hardware (for example, your computer) it generally cannot fulfill its safeguarding function.

Thus, it is axiomatic that even when using matter-energy boundaries to control matter-energy flows, information processing is required. Conversely, even when using information boundaries to control information flows, matter energy is required. To summarize, it takes matter-energy to process information, and information to process matter-energy. Some possible scenarios for boundary control and maintenance are shown in Table 1.

The classical distinction is that thermodynamics systems are "isolated" systems, whose boundaries are closed to flows of both energy and information (Bertalanffy, 1968). Thus, such an isolated system would occupy position number 1 in Table 1. No living system such as a social system could conceivably exist in such a permanentlyclosed fashion. Thus, in contrast to isolated or closed systems, social systems are said to be "open systems" (Bertalanffy, 1968; Parsons, 1951).

However, this term has often been used without clarification, implying that all social systems are permanently open. Often the types of flows that social systems are supposedly open to are not specified. This would imply that all social systems occupy position number 4 in Table 1 . While it is doubtful that any social system could survive if it remained permanently closed, it is theoretically possible (but highly unlikely) that a social system could exist in a permanently open state. However, this would certainly not provide an optimal quality of life. As we have indicated previously, a social group with no security measures in place to bar nonmembers can suffer not only losses such as the theft of resources, but also dilution of group identity, and contamination of its information base or ideology. Perhaps the sort of social 
group most likely to survive with totally open boundaries is one with minimal resources, and minimal invitations, theft, or contamination attractions.

Thus, most social groups are more likely to occupy either positions $2,3,5,6,7,8$, or 9 of Table 1. Note that Table 1 distinguishes between inflows or input (imports) and outflows or outputs (exports) of matter-energy and information respectively. Table 1 does not present so-called "through-puts", as these can generally be analyzed as consecutive input-output chains. Another possibility is an output that is immediately balanced, negated, or "canceled-out" by a subsequent consecutive input (or an input that is negated by an output). Again, this is not specifically represented in Table 1, but can be analyzed as a series of inputs and outputs.

Perusal of Table 1 quickly reveals that most contemporary societies, regardless of size and degree of complexity, fall roughly into Model number 9, indicating that they vary in terms of boundary closure for both inputs and outputs, and for both matter-energy and information. The average society cannot leave itself perpetually open even to flows of potentially valuable raw materials or food or energy, as it would then face subsequent problems of transportation and storage. It cannot leave itself open to unregulated information inputs without danger of information overload, which can be a rather severe problem (Miller, 1978). Similarly, it cannot leave itself perpetually open to exports of matter-energy or information without the risk of having all of its needed resources diminished. Thus, most societies fall roughly into Model number 9. However, this does to mean that the remaining six Models, numbers 2, 3, 5, 6, 7, and 8 , are devoid of theoretical or empirical interest. We will review them briefly, before returning to an in-depth analysis of Model number 9.

Model number 2, always open to inputs, and always closed to outputs, is probably rarely found empirically in social groups for rather obvious reasons. In Model number 2 of Table 1, doubtless some societies would be willing to receive inputs of goods without reciprocating through exportation, but this is obviously difficult to achieve in a world of shortages. Probably the contemporary society that comes closest to this is North Korea, as it lacks a market economy, and can afford comparatively few exports, as the goods of the sort it produces are generally needed at home. However, it does welcome inputs in the form of humanitarian food aid, and does receive such aid from a number of countries. Of course, many other societies that are experiencing some catastrophe may be the recipients of food aid and other aid that they are unable to reciprocate.

Model number 3 of Table 1 (matter-energy), this represents a social group that refuses imports, but constantly exports. Model number 5 is similar. It represents a society that is closed to imports, but varies as to whether it exports. Both numbers 3 and 5 would probably be rather wealthy societies that are relatively self sufficient, and thus do not have a pressing need for imports, but wish to export goods for cash. Model number 6 of Table 1 represents a society that never exports, but sometimes imports. This would likely be a rather poor society with little to export, but open to imports of goods that it needs (when this can be arranged without reciprocal exports). Similarly, Model number 7 could represent a relatively poor society with little to export, but that is more receptive to inputs than to exports. Model number 8 is again similar to Model number 3 , and would most likely represent a rather wealthy society with a lot of exports, but less receptive to imports than to exports. 


\section{Boundary Maintenance in LST and Social Entropy Theory}

As indicated above, Model number 9 of Table 1 is clearly where the bulk of social groups in the contemporary complex society lie. This is true for all hierarchical levels, from individuals through groups, organizations, institutions, and societies. These social systems monitor their boundaries, as well as their internal needs and desires, and then make decisions about whether to open or shut the boundary to imports, or to open or shut the boundary to exports. This applies, respectively, to both matter-energy and information. To be even more specific, it applies respectively to: (1) population (matter-energy); (2) internal environment (matter-energy); (3) external environment (matter-energy); (4) energy resources (matter-energy); (5) culture (information); (6) technology (matter-energy and information); (7) information (information); (8) organization (matter-energy and information); and (10) level of living (matter-energy). However, even though this book is about complex sociology, it is probably unnecessarily complex to analyze boundary control for all of these ten dimensions at this time. For the present, it is sufficient to simply acknowledge that all ten of these flows can be controlled individually (or in combination) at the boundary. The exact manner in which this is accomplished can be saved for future analysis. For now, we will only analyze boundary control for the broad categories of matter-energy and information.

In Table 1, most social systems fall into Model number 9, as noted previously. This means that they must constantly monitor flows of matter-energy into and out of their internal system. They need to maintain optimal levels of matter-energy (for example, raw material food, gas, and electricity). If they run short, they cannot conduct the daily work necessary for the maintenance of the social system, and their level of living may quickly decline. Conversely, if they have an unwanted surplus of these crucial matter-energy supplies, they will experience increasing time and money costs related to storage, transportation, spoilage, pest control, environmental impact, and so forth.

The frequency of monitoring and response of course depends upon the particular type of matter-energy. In terms of inflows (imports), "always on" resources such as electricity must be constantly monitored on a continuous basis. Increasingly, this monitoring of such things as electricity grids must be done by complex computer systems which unfortunately can be quite susceptible to computer "hackers", who can conceivably shut down the power for a whole state or region if computer security is lax.

When such "always on" systems are interrupted, response must be rapid. Other physical material or energy sources may require less monitoring and a less rapid response, even in the case of imports. While dealing with outflows (exports), these are less crucial for the internal system where they originate, and so generally monitoring and response can be slower (unless of course a trade partner has an urgent need for the resource that one is exporting to them).

We have previously been discussing matter-energy inflows and outflows across social-system boundaries. We will now conduct a similar analysis for information inflows and outflows. It is rare for a social system, regardless of hierarchical level, to be permanently closed to both inflows and outflows of information (Model 1). To speculate, a small social system (a single individual, for example), might be able to 


\section{Boundary Maintenance in LST and Social Entropy Theory}

close off most outflows of communication permanently (for example, by having no telephone or computer, and never mailing anything). It is more difficult to cut off incoming mail and other inflows, but this probably could be done. Ironically, individuals who purport to never watch television or listen to radio, or read newspaper, and thus have no knowledge of a murder, are the ones most coveted for juries, as they are thought by the media to be unbiased. Thus, the most detached and uninformed person is seen as the most desirable for jury duty.

It is doubtful that even an individual or family could totally eliminate all incoming and outgoing communications (except possibly in a closed institution such as a prison or asylum). However, it is clear that isolated groups living in the wilderness could come close to doing so (with relatively few exceptions, such as mandatory contact with the Internal Revenue Service). This becomes much more difficult at higher hierarchical level such as societies. While North Korea may be able to squelch most information from the West to most citizens, even it probably monitors some incoming information flows, and has some outflows (although carefully monitored) as well.

Models number 2 and number 3, with closed inputs and open outputs, or vice-versa, are empirically possible, but impractical. Perhaps Model number 2 is more feasible than Model number 3 for an individual, but both are impractical for larger systems such as societies. However, at the individual level, it is possible for a person to constantly monitor incoming information (as in the case of a criminal listening to police radio frequencies) but virtually never send outgoing information. However, the converse, where a person constantly sends out information, but never receives any, seems nearly logically impossible. It is possible in the case of a propaganda machine which wishes no information from the enemy, but merely wishes to disseminate propaganda 24-hours a day.

However, at the societal level, it is unlikely that a society could permanently shut down either incoming or outgoing information flows (Models number 2 and 3 respectively), just as we have already indicated the difficulty of shutting down both simultaneously (Model number 1). Models number 6 and 7 are very similar to Model number 2, so the comments made for Model number 2 tend to apply to Models number 6 and 7 as well. Similarly, Models number 5 and 8 are similar to Model number 3, so the comments previously made for Model number 3 apply to Models number 5 and 8 as well.

This leaves only Models number 4 and number 9, and both of these deserve more extensive analysis. At first glance, it would seem that while Model number 4 is clearly impractical for matter-energy flows, (Table 1, as previously discussed), it is more practical for information flows. That is, do not important lines of communication need to be on at all times, especially at higher hierarchical levels, for example, regarding vital issues of societal security? Obviously, one cannot shut off an incoming communication line, lest one miss an emergency message of some sort.

However, one cannot leave a line continually open if that line is subject to information overload. Such "information overload" can render a communication line almost as worthless as if it were closed completely. For extended discussion of information processing and information overload see Miller (1978). One mechanism for dealing with this is to have multiple communication lines. One can be left 


\section{Boundary Maintenance in LST and Social Entropy Theory}

permanently open, both for inflows and outflows, and thus fits Model number 4. However, the other more mundane lines are subject to information overload. These are flexible lines, and can be monitored closely, and closed to both inflows and outflows when desired.

Moving now to Model number 9, it is clear that most information lines are variable (flexible), both for inputs and outputs. This means generally that they are capable of being open for in and out transmissions at all times (generally with a minimum amount of effort), but can be closed when not needed or wanted. Examples of variable information lines abound. The most common include televisions, radios, telephones, computers, and hand-held devices such as two-way pagers. These devices can be left open constantly, or can be opened and closed quickly. The average social system has many of these communication lines. Probably the most common pattern is to have multiple lines that are open and closed depending upon need. These are augmented by one or a few "always on" lines such as facsimile (FAX) machines, telephone message machines, and computerized electronic mail.

I call these latter "partially always on" machines. This means that while they are always open to incoming transmission (up to their storage capacity), these transmissions do not have to be constantly monitored. Thus, one can let E-mail, telephone messages, and FAX messages accumulate to a certain point without continually monitoring them. However, if such messages are never monitored, they will eventually reach storage limits (storage capacity), and thus no more will be received until some of the ones received and stored earlier are eliminated.

To summarize, regarding both inflows and outflows of both matter-energy and information, all social systems at all hierarchical levels have by necessity some degree of monitoring and control over all types of flows. They must control flows across their external boundary, as well as internal flows. Many of them may also desire to control external flows that lie wholly in the external environment outside of their boundaries, but this of course can be much more difficult to accomplish. However, as we have seen, social systems vary widely in the types of flows, and how they are monitored and controlled. We may offer a few generalizations at this point. While social systems are often said to be "open systems", in reality most of them occupy Model number 9 in Table 1, meaning that they are not "always on", but are alternatively open and closed with regard to inflows and outflows of matter-energy.

It should be noted that many social groups have multiple parts, and so may differ in their control of these parts. That is, they may have one door open and another shut, or direct flows from one to another. But they are less likely than for information flows to be "always on" in terms of matter-energy flows. Turning to information flows, again most social systems, regardless of hierarchical level, fall in Model number 9, variables (alternatively open or closed) on both information inflows and outflows. However, more than for matter-energy, some groups may be "always on" or "always open" (Model number 4). The average social group is likely to have more information lines than matter-energy lines, and to have more of these "always open" (particularly emergency line). However, "always open" lines can quickly cause crises of information overload and unmanageability. Thus, the average social system is a mix of flexible (variable) lines, and "always open" lines, with the former predominating. 


\section{Boundary Maintenance in LST and Social Entropy Theory}

The chief characteristics of information processing compared to matter-energy processing, is that the former is much quicker, perhaps instantaneous. Thus, groups must react much more quickly to crises. Thus, social groups are more vulnerable to information crises, and must monitor information lines more closely. Social groups may possess a large number of information links. Information may be directed from one to another, often at very fast speeds. Some links may be kept open, with others kept closed, and others remaining more variable (alternatively open and closed). Also, devices such as Internet "routers" may quickly switch information from one line to another.

\section{INFORMATION INFLOWS}

Table 2 shows the effects of information inflows on social systems. Inputs can be neutral, harmful, or beneficial. A neutral inflow has no noticeable effect on the system. Harmful effects can range from mild (innocuous) to moderate to fatal. Among the very harmful or even fatal inputs are stolen intellectual property that makes an institution liable for damages, fatal viruses that destroy computer systems, faulty information that leads to a ruinous business decision, and so forth.

Another type of input is a canceling input. This is a qualitative input (either harmful or helpful), that offsets, cancels, or neutralizes another polar-opposite input of the same strength. Beneficial inputs can range from mildly helpful to so dramatic that they change the entire nature of the social system. Some inputs are not by themselves capable of changing the system, but can do it with the help of other inputs. Thus, each alone is a "partial change" agent. Such partial changes can either be beneficial or harmful (but by definition not neutral) to the system. These include necessary, but not sufficient inputs. Such an input is necessary for change in the system to occur, but cannot alone transform the system. For example, if input A is necessary, but not sufficient, this means that it "solves part of the puzzle", or is part of the solution that the system needs to help transform it.

Yet another type of information input, as shown in Table 2, is a contingent inflow. This is an input whose effect is somewhat indeterminate. It varies, depending upon the existence of another input. That is, the impact of the information inflow on the social system is dependent upon the existence and nature of another variable (or variables). Such contingency is an indication of non-linearity, bringing up another distinction. Another type of information input as listed in Table 2 may be described as a "catalytic". This is information that, either because of its timing or nature of the message (or both) becomes "the straw that broke the camel's back", or the catalyst that induces change in the social system that is the recipient of the information. 
Table 2. Types of Information Inflows Across Social Group Boundaries

1. Neutral

2. Moderately Helpful

3. Helpful

4. Slightly Harmful (Innocuous)

5. Fatal

6. Canceling (Two Messages Neutralize or Cancel Each Other).

7. Necessary (But Not Sufficient) For Change

8. Sufficient (But Not Necessary) For Change

9. Contingent

10. Catalytic

11. Linear

12. Nonlinear

13. False signal

14. Noise

As shown in Table 2, the information received can either be linear or nonlinear (curvilinear). The former indicates that the degree of societal change is constant at different levels for the information received. The latter (non-linearity) shows that the information has a different impact in terms of change in the social system depending upon the level of the variable (information)..

Yet another type of information input is the false signal. This is a misleading information input that falsely signals the social system to institute change, or to embark upon a particular course of action. For example, suppose that a large organization (business) is debating whether or not to invest a large amount of money in very expensive new equipment containing the latest (but relatively unproven) technology. The decision-makers rely upon an information input in the form of a certain economic statistic for the most recent month to help them make their final decision, as the deadline for ordering the equipment is nearing.

Occasionally, for one reason or another, the monthly economic figure will be an aberration, and will not be a valid indicator of the long-term trend. If the social system relies on such "false" information to aid in its decision making, it may well make an unwise decision, thus negatively impacting the organization for some time to come. The last sort of information input listed in Table 2 is "noise". This is when an input is received, but the information is regarded as extraneous or irrelevant, or there is so much correlated confusion or ambiguity surrounding the content of the message, that the reliability of the message, or even its very meaning, may be in doubt.

\section{INFORMATION OUTFLOWS}

In general, information outflows from a social system are basically just the reverse of inflows, and the same sort of analysis should suffice. Thus, a quick perusal of Table 3 helps analyze information outputs. Like inputs, outputs can be neutral, beneficial, 


\section{Boundary Maintenance in LST and Social Entropy Theory}

harmful, and so forth, for the social system. Also, coding (encoding in Miller's terms) for information outflows is essentially the reverse of the process for inflows, and all of the same problems are encountered, but in reverse (Bailey, 2007a, 2007b). Instead of switching from generic to private language as required for inflows, the message now must be switched from private to generic (public) language. Errors can be made if the generic language simply does not properly represent the full meaning inherent in the original private code, or if the insider coder does not have an adequate command of the generic public code. Unfortunately, there is again no assurance that the output message is a valid reflection of the original intent.

Thus, as with inflows, it should be assumed that the coding process for outgoing messages is inherently problematic. This does not mean that the messages are necessarily flawed. It simply means that one should not make a blanket assumption that coding can be done with total adequacy. We should recognize that coding, whether for inflows or outflows, is error prone, but is too important to be ignored in social analysis, as sociologists have frequently done.

In addition to the generic problems detailed in Table 3, information outflows pose some specific problems not prominent for inflows. These are detailed in Table 3. Table 3 classifies information outflows as being obligatory, optional, important, routine, long term, or short term. Obligatory flows include those mandated by external sources. These include such outflows as reports (sometimes quarterly or annual) that are required by entities higher in the social hierarchy.

\section{Table 3. Types of Information Outflows Across Social Group Boundaries}

1. Obligatory (Mandated reports)

2. Optional

3. Important

4. Routine

5. Long Term

6. Short Term

7. Formatted

8. Nonformatted

Other sorts of obligatory information outflows include messages vital to the well being of the social system, such as "May Day" or "911" emergency calls for aid. In addition to the obligatory or mandated information outputs, social systems output many messages which are optional, such as press releases, letters, and so forth. Many of these are not required, but are simply designed to create a positive impression, to continue or begin a relationship, or in general to benefit the sender by increasing the well being of the social system.

Social systems may also classify their information outputs as important or routine. Many obligatory messages such as government reports may be considered 


\section{Boundary Maintenance in LST and Social Entropy Theory}

unimportant (but required) by the organization. Other outputs may be important in either a positive or negative sense. This parallels the harmful, helpful, and neutral types of input discussed in Table 2. Information outputs also may be either long term (even permanent) or short term (temporary, or perhaps consisting of only a single occurrence). Some of the obligatory reports, for example, may be essentially permanent. Other outgoing messages may only occur once, and may never need to be repeated.

Outgoing messages also vary in terms of format. Some reports required by outside agencies may dictate a set format without any deviation permitted. Other messages may have a more flexible format. Sometimes the format, such as message length, font size, margin, and so forth, may be dictated by the receiver or by the sender. Other times it may be dictated neither by the sender or receiver, but instead by the particular information technology used, such as computer hardware, software, E-mail systems, and so forth.

\section{MATTER-ENERGY INPUTS AND OUTPUTS}

Matter-energy (M-E) flows differ from information flows in some very basic ways. M-E flows tend to be slower and bulkier, and may take longer to process than information flows. While they do not require de-coding in the same way that information does, they may require considerable processing or transformation before they can even be utilized by the equipment (such as the manufacturing machinery) of the social system.

As shown in Table 4, roughly the same types of inflows and outflows may occur for M-E as for information outflows. That is, they may be classified as obligatory outflows that are not optional, such as the outputting of toxic wastes), or optimal. They may also be vitally important in either a positive or negative sense (crucial to the survival of the social system), or rather routine and largely unimportant. They also may be long-term, even permanent flows, or temporary, every unique or one-time events.

\section{Table 4. Types of Matter-Energy Inflows and Outflows}
1. Obligatory
2. Optional
3. Important
4. Routine
5. Long Term
6. Short Term

Perhaps the biggest difference between handling M-E flows and information flows is that the former are larger, bulkier, and more difficult to process. They may be slower to receive, and slower to process. Problems with flows also may be more difficult to rectify. For instance, an error in an incoming information message may be corrected 


\section{Boundary Maintenance in LST and Social Entropy Theory}

via a single E-mail. In contrast, an error in an M-E inflow, such as the receipt of 100 tons of the wrong kind of ore, may be costly and time-consuming to correct.

\section{CONCLUDING REMARKS}

All inflows and outflows, whether of M-E or information, and regardless of the level of the social group (group, organization, society) all have a variety of problem s and features that we cannot deal with here. These include problems of processing, storage, coding, and so forth.

The full discussion of such issues is beyond the scope of this paper. Our point here is not to exhaustively discuss these issues, but to merely repair some of the extreme neglect of these issues. This neglect has resulted from the tendency of social-systems theorists to study the internal features of social groups, while neglecting boundary problems, inflows and outflow, and relationships with other groups, and with the environment as a whole.

The next step is to go beyond the analysis of boundary actions, to the full study of flows between systems. Further exegesis is found in Bailey $(2005,2006)$, and Swanson and Bailey, (2006). The increasing complexity of future societies, along with electronic advances, will ensure an impressive array of information flows of various sorts, both intra-systemic and inter-systemic. The challenge to social-systems theorists is to conduct a comprehensive analysis of such flows without neglecting the analysis of matter-energy flows, which remain an important aspect of complex social systems.

\section{REFERENCES}

Bailey, K. (1990). Social Entropy Theory, State University of New York Press, Albany.

Bailey, K. (1994). Sociology and the New Systems Theory, State University of New York Press, Albany.

Bailey, K. (1997). "The Autopoiesis of Social Systems: Assessing Luhmann's Theory of Self-Reference," Systems Research and Behavioral Science 14 (2): 83-100.

Bailey, K. (2005). "Beyond System Internals: Expanding the Scope of Living Systems Theory," Systems Research and Behavioral Science 22(6): 497-508.

Bailey, K. (2006). "Living Systems Theory and Social Entropy Theory," Systems Research and Behavioral Science 23: 291-300.

Bailey, K. (2007a). "Public and Private Codes in Living Systems Theory," Systems Research and Behavioral Science, forthcoming.

Bailey, K. (2007b). "Insider Coding: Congruence in the Theories of Luhmann and Miller," Journal of Sociocybernetics, forthcoming.

Bertalanffy, L. v. (1968). General System Theory, George Braziller, New York. Luhmann, N. (1995). Social Systems, Stanford University Press, Stanford, CA. Miller, J. (1978). Living Systems, McGraw-Hill, New York. 


\section{Boundary Maintenance in LST and Social Entropy Theory}

Miller, J.L., and Miller, J.G. (1992). "Greater Than the Sum of its Parts-I: Subsystems Which Process Both Matter-Energy and Information," Behavioral Science 37: 1-38.

Parsons, T. (1951). The Social System, Free Press, Glencoe, IL.

Swanson, G.A., and Bailey, K. (2006). "Social Entropy Theory, Macro Accounting, and Entropy Related Measures." In Proceedings of the Annual Meeting of the International Society for the Systems Sciences, (J. Wilby and J. Allen eds.). Sonoma, CA. 